\title{
Evaluation of Clinical Interest of Anti-Aquaporin-4 Autoantibody Followup in Neuromyelitis Optica
}

\author{
Jean-Baptiste Chanson, ${ }^{1,2}$ Melissa Alame, ${ }^{3}$ Nicolas Collongues, ${ }^{1,2}$ Frédéric Blanc, ${ }^{1,2}$ \\ Marie Fleury, ${ }^{1}$ Gabrielle Rudolf, ${ }^{1,2}$ Jérôme de Seze, ${ }^{1,2}$ and Thierry Vincent ${ }^{3,4}$ \\ ${ }^{1}$ Département de Neurologie, Hôpitaux Universitaires de Strasbourg, 1 Avenue Molière, 67091 Strasbourg, France \\ ${ }^{2}$ Laboratoire d'Imagerie et de Neurosciences Cognitives (LINC), Université de Strasbourg-CNRS, 1 rue Kirschleger, \\ 67000 Strasbourg, France \\ ${ }^{3}$ Département d'Immunologie, Hôpital Saint-Eloi, Centre Hospitalier Universitaire de Montpellier, \\ 80 Avenue Augustin Fliche, 34295 Montpellier, France \\ ${ }^{4}$ Institut de Génétique Humaine, CNRS UPR1142, 141, rue de la Cardonille, 34396 Montpellier, France \\ Correspondence should be addressed to Thierry Vincent; t-vincent@chu-montpellier.fr
}

Received 24 December 2012; Accepted 2 April 2013

Academic Editor: Y. Yoshikai

Copyright (C) 2013 Jean-Baptiste Chanson et al. This is an open access article distributed under the Creative Commons Attribution License, which permits unrestricted use, distribution, and reproduction in any medium, provided the original work is properly cited.

\begin{abstract}
Neuromyelitis optica (NMO) is an autoimmune disease in which a specific biomarker named NMO-IgG and directed against aquaporin-4 (AQP4) has been found. A correlation between disease activity and anti-AQP4 antibody (Ab) serum concentration or complement-mediated cytotoxicity has been reported, but the usefulness of longitudinal evaluation of these parameters remains to be evaluated in actual clinical practice. Thirty serum samples from $10 \mathrm{NMO}$ patients positive for NMO-IgG were collected from 2006 to 2011. Anti-AQP4 Ab serum concentration and complement-mediated cytotoxicity were measured by flow cytometry using two quantitative cell-based assays (CBA) and compared with clinical parameters. We found a strong correlation between serum anti-AQP4 Ab concentration and complement-mediated cytotoxicity $(P<0.0001)$. Nevertheless, neither relapse nor worsening of impairment level was closely associated with a significant increase in serum Ab concentration or cytotoxicity. These results suggest that complement-mediated serum cytotoxicity assessment does not provide extra insight compared to anti-AQP4 Ab serum concentration. Furthermore, none of these parameters appears closely related to disease activity and/or severity. Therefore, in clinical practice, serum anti-AQP4 reactivity seems not helpful as a predictive biomarker in the followup of NMO patients as a means of predicting the onset of a relapse and adapting the treatment accordingly.
\end{abstract}

\section{Introduction}

Neuromyelitis optica (NMO) is a severe inflammatory demyelinating disease of the central nervous system in which inflammatory lesions are usually restricted to the spinal cord and optic nerves $[1,2]$. NMO is distinguished from multiple sclerosis (MS) by a more severe evolution and a predominant humoral response [3]. A highly disease-specific serum antibody $(\mathrm{Ab}), \mathrm{NMO}$ immunoglobulin G (NMO-IgG), has been recently discovered [4]. The target antigen of NMO-IgG was identified as aquaporin-4 (AQP4), the main water channel protein in the CNS [5]. Owing to its very high specificity in $\mathrm{NMO}$, anti-AQP4 $\mathrm{Ab}$ was included in the revised diagnostic criteria for NMO [6]. Anti-AQP4 Ab also proved to be helpful in predicting a more severe course and a probable conversion to NMO after a first episode of isolated optic neuritis or longitudinally extensive transverse myelitis [711]. Several experiments in animal models demonstrated a pathogenic role of this antibody in the disease [12-14]. Thereafter, it was questioned whether anti-AQP4 Ab serum concentration is related to disease activity in humans and, thus, whether its assessment could be helpful in predicting disease evolution and adapting the treatment.

A link between anti-AQP4 serum reactivity and various clinical parameters in NMO patients was suggested by numerous studies. Takahashi et al. found an association 
between high anti-AQP4 Ab serum concentration and both transverse myelitis lesion length and disease activity [15]. Accordingly, Jarius et al. [16] and Waters et al. [17] described increased anti-AQP4 serum concentration during clinical relapses and a decrease following immunosuppressive therapy. Kim et al. suggested that anti-AQP4 Ab levels correlate with disease activity but noted that rising of anti-AQP4-Ab levels did not always lead to acute exacerbation [18]. However, when testing complement-dependant cytotoxic properties of NMO patients' serum on AQP4 transfected cells, Hinson et al. observed that severe relapses were associated with higher cytotoxicity but not higher anti-AQP4 serum concentration [19]. Finally, Dujmovic et al. found that cerebrospinal fluid (CSF) but not serum anti-AQP4 Ab titers are associated with disease activity and neuroinflammation [20], whereas Jarius et al. suggested that both parameters are correlated with relapses [21].

Here, we aimed to evaluate the interest of a longitudinal assessment of anti-AQP4 Ab in clinical practice. We therefore compared the evolution of both anti-AQP4 Ab serum concentration and complement-mediated cytotoxicity with key clinical parameters such as relapse onset and disease severity.

\section{Patients and Methods}

2.1. Patients. Among the NMO patients followed up in the Neurology Department of the University Hospital of Strasbourg, we selected those who were positive for NMO-IgG. The disease was diagnosed according to the revised criteria of Wingerchuk et al. [6]. NMO-IgGs were detected by indirect immunofluorescence (IIF) on primate cerebellum slides with diluted sera (1/50) following the manufacturer's instructions (Instrumentation Laboratory, Lexington, MA, USA). Patients must have presented at least one relapse in the 5 last years. Ten NMO patients were included. We collected both retrospectively and prospectively serum samples taken during a relapse or a control visit. Presence of a relapse and disease severity (evaluated by the Expanded Disability Status Scale, EDSS) at the time of the blood draw were registered. Relapses were treated at least with intravenous high-dose methylprednisolone (1000 mg/day over 3-5 days) and for the most severe relapses with plasma exchanges (PE, 4-5 PE cycles). The samples were taken before corticosteroid treatment or PE. The interval between the onset of the relapse and the blood draw was from three to seven days. Presence of an immunosuppressive therapy (cyclophosphamide, mycophenolate mofetil, azathioprine, mitoxantrone, or rituximab) was also registered. Written informed consent was obtained from all patients. Study protocol was declared to the CNIL (Commission Nationale Informatique et Libertés), and the study was performed in accordance with the ethical standards laid down in the 1964 Declaration of Helsinki.

2.2. Measurement of Anti-AQP4 Ab Serum Concentration. Sera were collected between 2006 and 2011 and stored at $-80^{\circ} \mathrm{C}$. They were sent to the Department of Immunology of the University Hospital of Montpellier (France). Anti-AQP4 $\mathrm{Ab}$ serum concentration was measured without knowledge of clinical information using a new quantitative cell-based assay (CBA) recently validated [22]. Briefly, HEK-293 T human cell line was transduced using HIV-based vectors expressing either the human AQP4 and enhanced green fluorescent protein (EGFP) genes (293-AQP4/EGFP cells) or the EGFP gene alone (293-EGFP cells). Cells were incubated with diluted human sera (1/200) in FACS buffer (PBS containing $1 \%$ fetal calf serum and $0.1 \%$ sodium azide), washed, and incubated with allophycocyanin- (APC-)conjugated goat anti-human IgG Ab (Jackson ImmunoResearch Laboratories, West Grove, USA). After washes, cells were analyzed on a FACS Calibur (BD Biosciences), and APC-mean fluorescence intensity (MFI) was measured on EGFP positive cells. A serum was considered anti-AQP4 positive if it bound 293AQP4/EGFP but not 293-EGFP cells. To determine the antiAQP4 concentration (arbitrary units; AU/mL), the APCMFI of 293-AQP4/EGFP cells incubated with 1/200 diluted patient sera and corrected for the background binding to the 293-EGFP control cells was plotted on a calibration curve obtained with a pool of NMO-IgG positive sera diluted from $1 / 200(1000 \mathrm{AU} / \mathrm{mL})$ to $1 / 51200(3.9 \mathrm{AU} / \mathrm{mL})$. All sera from the same patient were analyzed simultaneously.

2.3. Measurement of Complement-Mediated Serum Cytotoxicity. The protocol was inspired from Hinson et al. [19]. 293-AQP4/EGFP and 293-EGFP cells were incubated with patients' sera (20\% serum in FACS buffer for 30 minutes at $\left.+4^{\circ} \mathrm{C}\right)$. After washes, cells were incubated with active complement $\left(40 \%\right.$ complement for 45 minutes at $37^{\circ} \mathrm{C}$; Low-Tox- $\mathrm{H}$ rabbit complement; Cedarlane Laboratories Ltd, Burlington, NC, USA) and complement-dependent cytotoxicity (CDC) was measured by flow cytometric analysis of the percentage of EGFP positive cells permeable to the viability dye propidium iodide (PI). A pool of normal sera from healthy subjects was included in each experiment as a negative control. Serum cytotoxicity was defined $(\mathrm{AU} / \mathrm{mL})$ by the difference between the percentage of PI positive cells induced in 293AQP4/EGFP cells versus 293-EGFP cells corrected by the background of mortality obtained with the pool of normal sera. All sera from the same patient were analyzed simultaneously.

2.4. Statistical Analysis. The Pearson correlation coefficient was used to search for a correlation between anti-AQP4 Ab serum concentration and cytotoxicity. The nonparametric Mann-Whitney $U$ test was used to determine whether antiAQP4 serum concentration or cytotoxicity was related to the presence of a relapse or disease severity. We compared both biological parameters in samples taken in relapsing phase ( $<1$ month after a relapse) versus remitting phase and in the 5 more disabled patients (i.e., with the highest mean EDSS scores) versus the other ones. A Bonferroni correction for multiple comparisons was applied and the statistical significance was defined as $P$ value of $P<0.01$.

\section{Results}

3.1. Patients. Thirty sera were collected from 10 NMO-IgG positive patients (mean: 3 per patient, range 2-5) between 2006 and 2011. Patients' characteristics are summarized in 
TABLE 1: Clinical features of NMO patients.

\begin{tabular}{lccccc}
\hline Patient number & Age $(\mathrm{yrs})$ & Disease duration $(\mathrm{yrs})$ & Follow-up duration $(\mathrm{mo})$ & EDSS & Immunosuppressive treatment \\
\hline 1 & 54 & 4 & 31 & 0 & AZT \\
2 & 19 & 2 & 37 & 2.5 & AZT \\
3 & 38 & 6 & 53 & 3.5 & MMF \\
4 & 61 & 1 & 30 & 3 & None \\
5 & 19 & 1 & 14 & 8 & AZT \\
6 & 63 & 12 & 6.5 & MTX then AZT \\
7 & 27 & 3 & 2.5 & 4 & RTX \\
8 & 46 & 0.1 & 0.5 & 5.5 & CYC \\
9 & 62 & 1.5 & 6 & 3.5 & PE \\
10 & 54 & 1 & $26.5 \pm 16$ & $3.4 \pm 3$ & AZT \\
\hline Mean \pm SD & $44.3 \pm 17.5$ & $3.2 \pm 3.8$ & &
\end{tabular}

yrs: years; mo: months; disease duration: duration from the first symptom of the disease to the first blood draw; follow-up duration: duration from the first to the last blood draw; EDSS: EDSS score evaluating disease severity; AZT: azathioprine; MMF: mycophenolate mofetil; RTX: rituximab; MTX: mitoxantrone; CYC: cyclophosphamide; PE: plasma exchanges.

TABLE 2: Relationship between anti-AQP4 Ab serum concentrations, complement-mediated cytotoxicity, and clinical parameters.

\begin{tabular}{|c|c|c|c|c|}
\hline & Anti-AQP4 concentration $(\mathrm{AU} / \mathrm{mL})$ & $P$ & Serum cytotoxicity (AU/mL) & $P$ \\
\hline Patients during a relapse & $124 \pm 127$ & \multirow{2}{*}{$P=0.71$} & $13.5 \pm 12$ & \multirow{2}{*}{$P=0.89$} \\
\hline Patients during a remitting phase & $176 \pm 224$ & & $14.2 \pm 11$ & \\
\hline Patients with the more severe impairment & $94 \pm 52$ & \multirow{2}{*}{$P=0.38$} & $10.2 \pm 7.7$ & \multirow{2}{*}{$P=0.21$} \\
\hline Patients with the milder impairment & $208 \pm 248$ & & $17 \pm 13$ & \\
\hline
\end{tabular}

Anti-AQP4 Ab serum concentration and complement-mediated serum cytotoxicity were measured in 30 sera from 10 NMO patients with a cell-based assay as described in methods. We compared both biological parameters in samples taken in relapsing phase ( $<1$ month after a relapse) versus remitting phase and in the 5 more disabled patients (i.e., with the highest EDSS scores) versus the other ones. $P$ : alpha risk.

Table 1. All were women. Ten samples (33\%) were taken during a relapse. The mean interval between two samplings was $10.7 \pm 8$ months (mean \pm standard deviation).

\subsection{Evaluation of Anti-AQP4 Ab Serum Concentration} and Complement-Dependent Cytotoxicity. As some studies described discrepancies between anti-AQP4 Ab serum concentration and complement-dependent cytotoxicity, we measured and compared these parameters in different NMO patients' sera. Mean anti-AQP4 Ab serum concentration was $158 \pm 136 \mathrm{AU} / \mathrm{mL}$ (range 132->1000) and mean cytotoxicity was $14.1 \pm 11 \mathrm{AU} / \mathrm{mL}$ (range $0-38.8$ ). We observed a high intra- and interindividual variability especially in anti-AQP4 $\mathrm{Ab}$ serum concentration. However, as shown in Figure 1, serum anti-AQP4 Ab concentration and complement-dependent cytotoxicity were highly correlated $(r=0.87, P<$ $0.0001)$.

\subsection{Relationships between Biological and Clinical Parame-} ters. Detailed anti-AQP4 reactivity course was compared to relapse onset, EDSS changes, and treatment for each patient as shown in Figures 2 and 3. As summarized in Table 2, we did not observe any significant correlation between biological parameters and disease activity. Indeed, increase in antiAQP4 Ab concentration may occur with no clinical change (e.g., patient 1), and conversely disease worsening was often not accompanied by an increase in anti-AQP4 Ab levels (patients 2 and 3). A significant effect of immunosuppressant was noted on anti-AQP4 Abs concentration. Indeed,

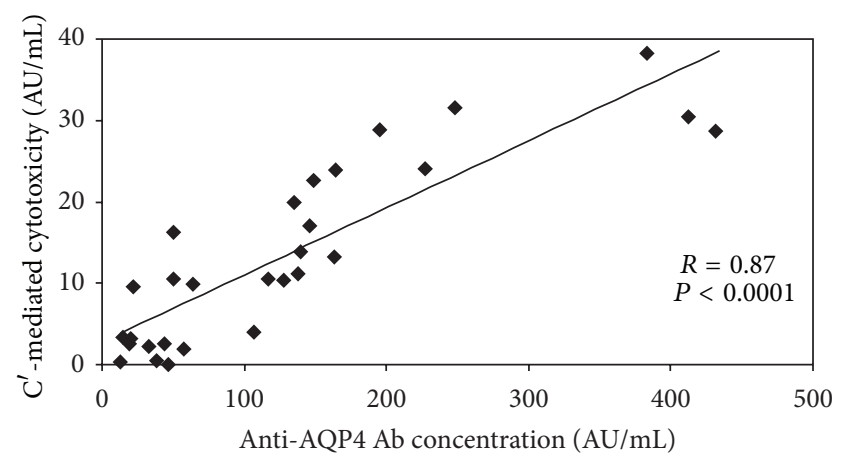

FIGURE 1: Correlation between serum anti-AQP4 Ab concentration and serum complement- ( $C^{\prime}$-)mediated cytotoxicity. Anti-AQP4 Ab serum concentration and complement- $\left(C^{\prime}\right.$-)mediated serum cytotoxicity were measured in 30 sera from $10 \mathrm{NMO}$ patients with a cellbased assay using AQP4 expressing human $293 \mathrm{~T}$ cells and flow cytometry. Results are expressed in arbitrary units $(\mathrm{AU} / \mathrm{mL})$. The Pearson correlation coefficient $(r)$ was $0.87(P<0.0001)$.

mycophenolate mofetil initiation was concomitant with a marked decreased anti-AQP4 reactivity in patient 3. A less significant decrease was observed under azathioprine in patient 2 and under cyclophosphamide in patient 6 . Plasma exchanges resulted in a rapid anti-AQP4 reactivity decline (patient 9). However, a similar effect was not observed under azathioprine in patients 1 and 6. Rituximab initiation in 


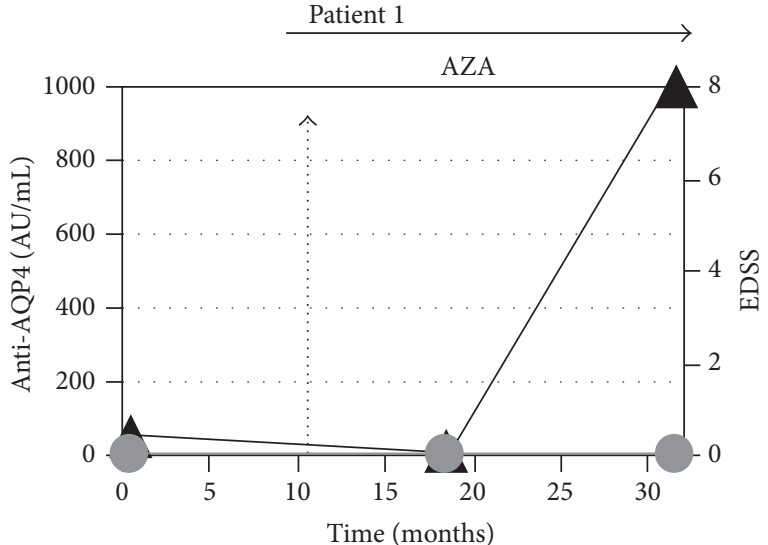

(a)

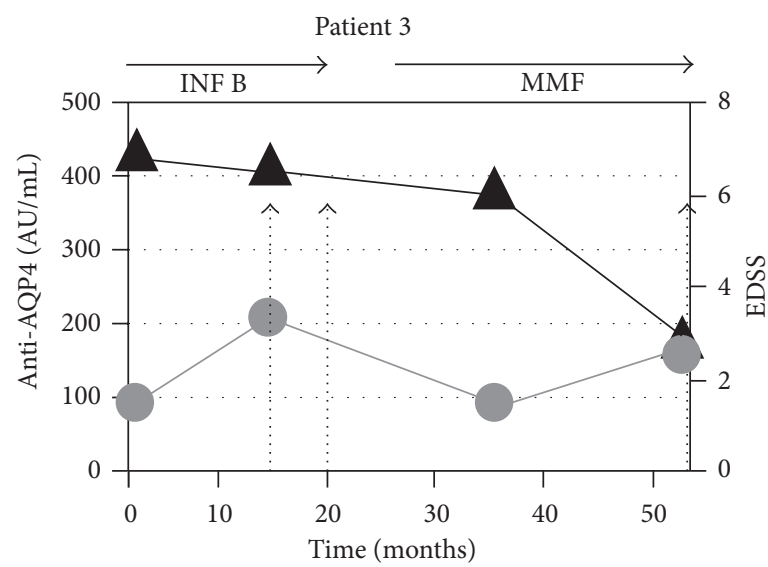

(c)

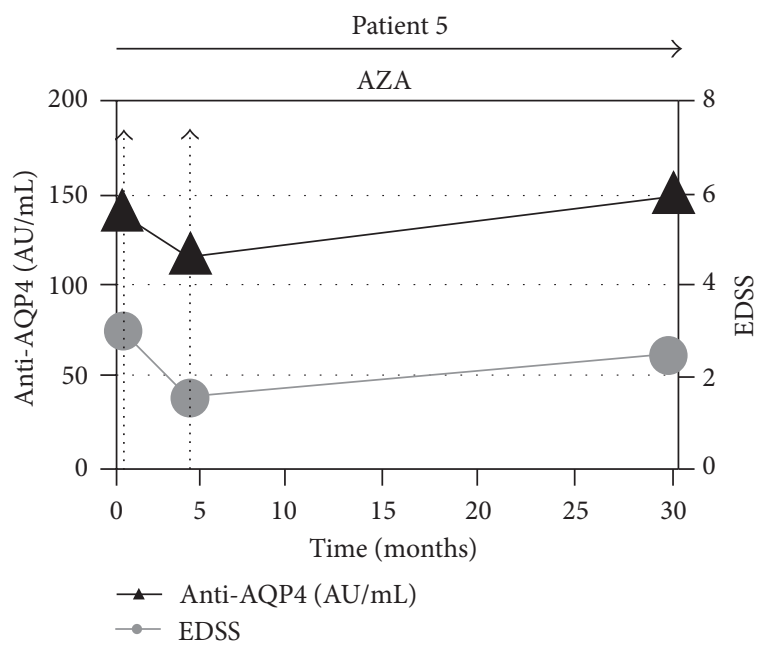

(e)

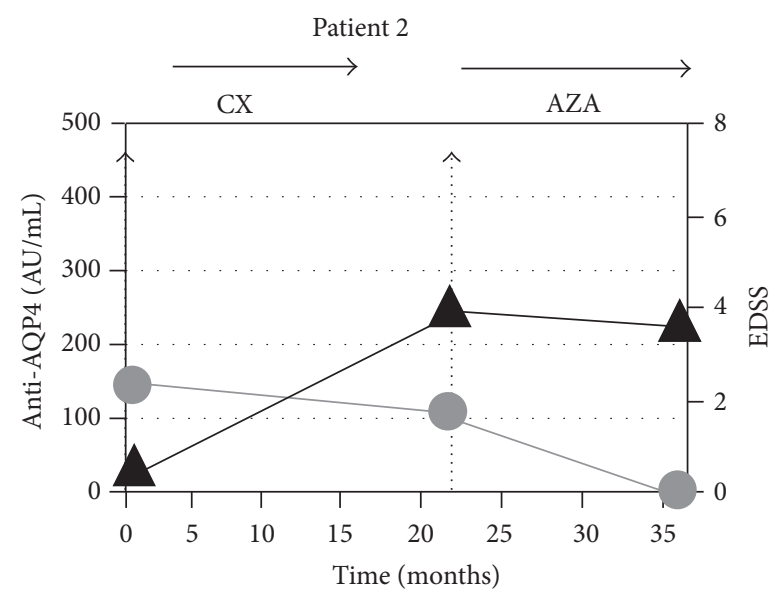

(b)

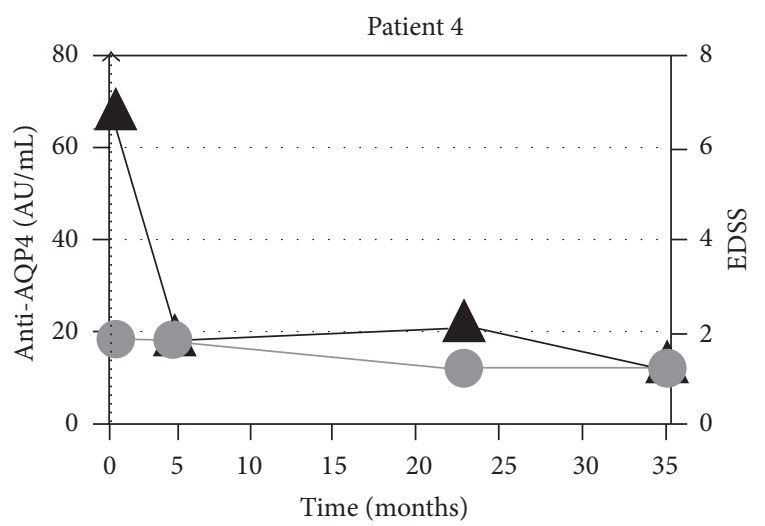

(d)

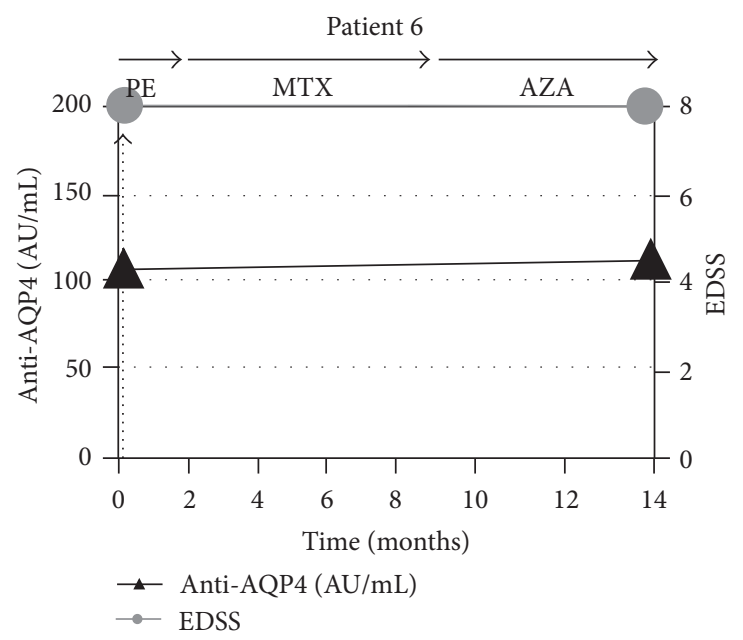

(f)

FIGURE 2: Detailed evolution of anti-AQP4 serum reactivity and comparison with disease course in patients 1 to 6 . Serum anti-AQP4 Ab concentration (black triangle) was measured with a cell-based assay using AQP4 expressing human 293 T cells and flow cytometry as described in Section 2. EDSS score indicates the level of impairment (grey circle); vertical dashed arrows represent relapses (all relapses were treated with corticosteroids); horizontal arrows indicate a treatment (AZA: azathioprine; CX: monthly administration of IV corticoids; INF B: interferon beta; MMF: mycophenolate mofetil; PE: plasma exchange; MTX: mitoxantrone). 


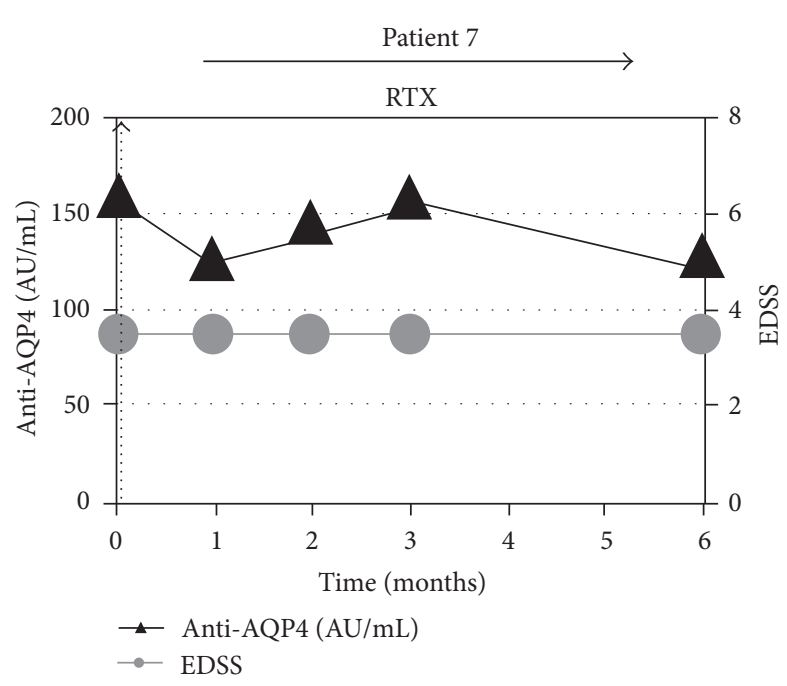

(a)

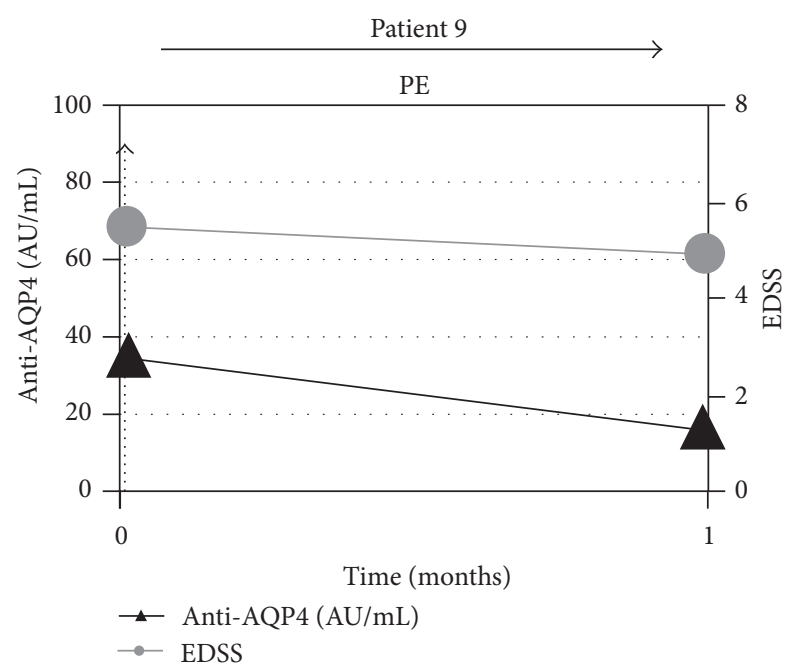

(c)

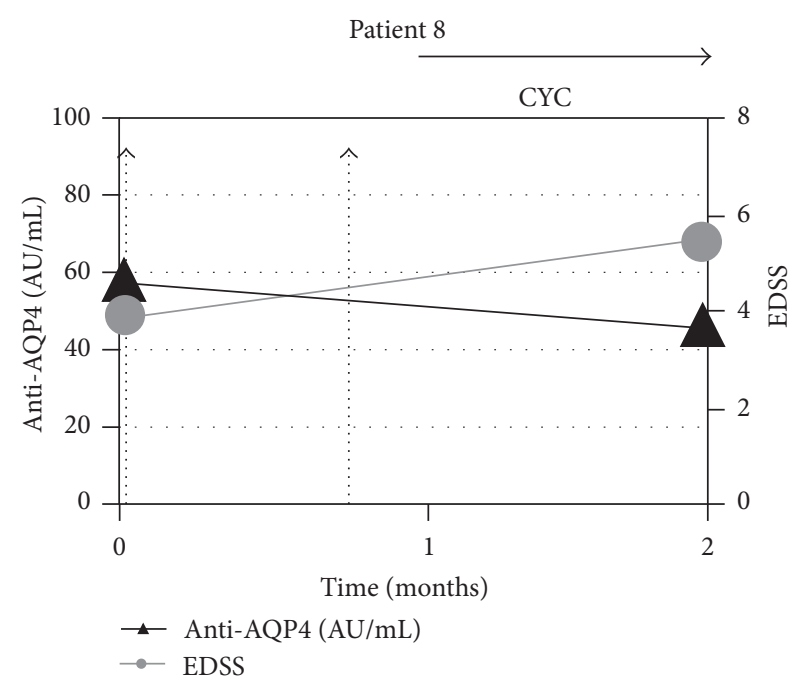

(b)

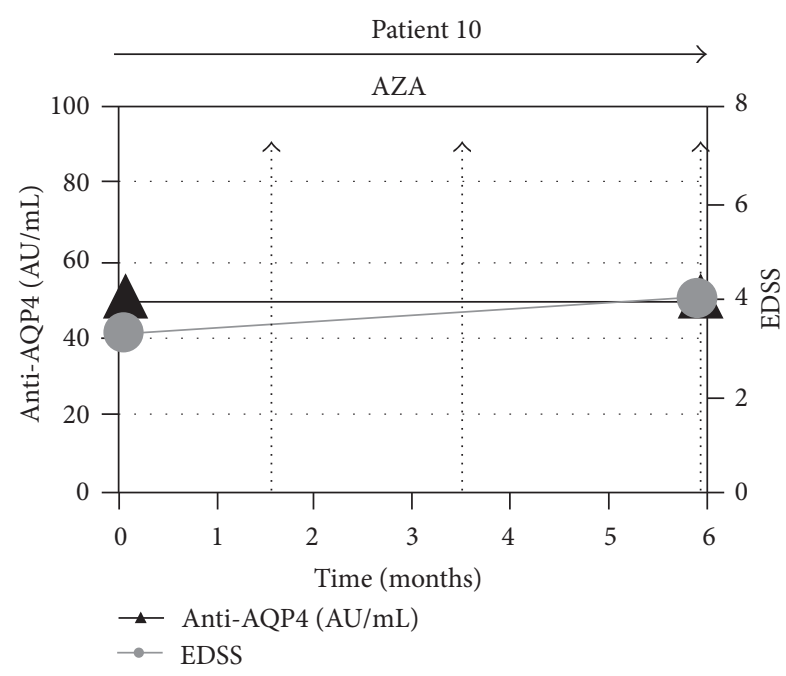

(d)

FIGURE 3: Detailed evolution of anti-AQP4 serum reactivity and comparison with disease course in patients 7 to 10 . Serum anti-AQP4 Ab concentration (black triangle) was measured with a cell-based assay using AQP4 expressing human 293 T cells and flow cytometry as described in Section 2. EDSS score indicates the level of impairment (grey circle); vertical dashed arrows represent relapses (all relapses were treated with corticosteroids); horizontal arrows indicate a treatment (RTX: rituximab (1 perfusion weekly of $375 \mathrm{mg} / \mathrm{m}^{2}$ during 4 weeks); CYC: cyclophosphamide; PE: plasma exchange; AZA: azathioprine).

patient 7 resulted in an early increase (with no clinical event) followed by a delayed decrease (at 6 months) in anti-AQP4 reactivity.

As shown in Table 2, statistical analysis found no relationship between anti-AQP4 serum concentration or complement-mediated cytotoxicity and the presence of a relapse or disease severity (EDSS score).

\section{Discussion}

The followup of our patients suggests that anti-AQP4 Ab serum concentration and complement-mediated cytotoxicity are strongly correlated, but that their monitoring does not allow an accurate and reliable prediction of disease evolution.

The only study that longitudinally assessed serum complement-mediated cytotoxicity in NMO patients reported that this parameter was better correlated with disease activity than anti-AQP4 Ab serum concentration [19]. These findings were not in accordance with the marked link previously demonstrated in vitro between anti-AQP4 Ab concentration and complement-dependent cytotoxicity [23, 24]. In our patients, despite high inter- and intraindividual variability, we found a marked correlation between anti-AQP4 Ab serum concentration and complement-mediated serum cytotoxicity 
$(P<0.0001)$. This result suggests that measurement of serum cytotoxicity does not give an extra insight compared to antiAQP4 Ab concentration assessment. Accordingly, when compared with disease course or severity, anti-AQP4 Ab serum concentration and complement-mediated cytotoxicity gave very similar and nonsignificant results.

NMO is characterized by severe relapses often resulting in sustained functional impairment and it would be of particular interest to predict a relapse onset for an earlier adaptation of the treatment [25]. Unfortunately, in the followup of our patients, relapses were rarely associated with relevant changes in anti-AQP4 reactivity. This is well illustrated by patients 2 and 3 in which anti-AQP4 reactivity was lower during a relapse than a remitting phase. Conversely, we observed in other cases that marked changes in anti-AQP4 reactivity were not accompanied by any clinical event (e.g., patient 1). A potential confounding factor was the presence of immunosuppressive therapies which are often prescribed in NMO $[15,16]$. We frequently observed a decrease in anti-AQP4 Ab concentration after the beginning of an immunosuppressive treatment. However, this effect was not systematic as was shown in patients 1 and 6. Transient increase in anti-AQP4 reactivity observed after Rituximab initiation in patient 7 is consistent with those reported in two recent papers $[26,27]$. Interestingly, these studies did not observe any correlation between the increase in anti-AQP4 Ab serum concentration and the worsening of the disease.

Our results do not preclude a pathogenic role of serum anti-AQP4 $\mathrm{Ab}$ in NMO but underline the requirement of additional factors to allow peripheral Abs to cross the bloodbrain barrier (BBB) and gain their targets in the CNS. Indeed, the worsening of experimental autoimmune encephalitis after intraperitoneal injection of anti-AQP4 Abs in animal was dependant on a previous $\mathrm{BBB}$ breakdown and the presence of inflammatory cytokines [12]. Likewise, intrathecal or intracerebral injection of NMO-IgG in the CNS induces NMOlike lesions, whereas peripheral injection does not cause CNS damage [14]. Indeed, although tolerance breakdown is thought to occur in the periphery, pathogenic processes occur in the CNS. Hence, as suggested by recent studies, it might be more relevant to use CSF rather than serum anti-AQP4 titer as a biomarker for the followup of NMO patients [20,28].

Our study presented some limitations that should be pointed out. The relative small number of patients tested may have limited the detection of significant correlations between immunological and clinical parameters. This study cannot address in detail the question whether relapses are preceded by an increase in AQP4 serum levels, since no paired samples taken shortly before and during relapse onset were available for comparison from most patients. Due to the retrospective design, time intervals between the samplings and treatments of patients were variable. The statistical results must be therefore interpreted with caution. However, this heterogeneity reflects the clinical practice in which patients are not monitored with short and regular intervals and receive various drugs. At an individual level, we noted that anti-AQP4 Ab concentration is highly variable and is related to many factors including the administration of immunosuppressants. However, it did not appear closely correlated with disease activity.

\section{Conclusions}

Altogether, our results showed that anti-AQP4 serum concentration in NMO patients is representative of serum complement-mediated cytotoxicity. The lack of strong correlation between the anti-AQP4 serum reactivity and disease course in our study suggests that anti-AQP4 Ab monitoring could not be used to reliably predict relapses and adapt treatment. Therefore, the longitudinal evaluation of serum anti-AQP4 reactivity does not appear helpful for an accurate followup of NMO patients in clinical practice.

\section{Conflict of Interests}

The authors have no financial conflict of interests to declare.

\section{Acknowledgment}

This study was supported by a grant from the CHU de Montpellier to T. Vincent.

\section{References}

[1] J. De Seze, C. Lebrun, T. Stojkovic, D. Ferriby, M. Chatel, and P. Vermersch, "Is Devic's neuromyelitis optica a separate disease? A comparative study with multiple sclerosis," Multiple Sclerosis, vol. 9, no. 5, pp. 521-525, 2003.

[2] D. M. Wingerchuk, V. A. Lennon, C. F. Lucchinetti, S. J. Pittock, and B. G. Weinshenker, "The spectrum of neuromyelitis optica," Lancet Neurology, vol. 6, no. 9, pp. 805-815, 2007.

[3] C. F. Lucchinetti, R. N. Mandler, D. McGavern et al., "A role for humoral mechanisms in the pathogenesis of Devic's neuromyelitis optica," Brain, vol. 125, no. 7, pp. 1450-1461, 2002.

[4] P. V. A. Lennon, D. M. Wingerchuk, T. J. Kryzer et al., "A serum autoantibody marker of neuromyelitis optica: distinction from multiple sclerosis," Lancet, vol. 364, no. 9451, pp. 2106-2112, 2004.

[5] V. A. Lennon, T. J. Kryzer, S. J. Pittock, A. S. Verkman, and S. R. Hinson, "IgG marker of optic-spinal multiple sclerosis binds to the aquaporin-4 water channel," Journal of Experimental Medicine, vol. 202, no. 4, pp. 473-477, 2005.

[6] D. M. Wingerchuk, V. A. Lennon, S. J. Pittock, C. F. Lucchinetti, and B. G. Weinshenker, "Revised diagnostic criteria for neuromyelitis optica," Neurology, vol. 66, no. 10, pp. 1485-1489, 2006.

[7] G. Akman-Demir, E. Tüzün, P. Waters et al., "Prognostic implications of aquaporin-4 antibody status in neuromyelitis optica patients," Journal of Neurology, vol. 258, no. 3, pp. 464470, 2011.

[8] S. Jarius, J. Frederikson, P. Waters et al., "Frequency and prognostic impact of antibodies to aquaporin- 4 in patients with optic neuritis," Journal of the Neurological Sciences, vol. 298, no. 1-2, pp. 158-162, 2010.

[9] M. Matiello, V. A. Lennon, A. Jacob et al., "NMO-IgG predicts the outcome of recurrent optic neuritis," Neurology, vol. 70, no. 23, pp. 2197-2200, 2008.

[10] B. G. Weinshenker, D. M. Wingerchuk, S. Vukusic et al., "Neuromyelitis optica IgG predicts relapse after longitudinally extensive transverse myelitis," Annals of Neurology, vol. 59, no. 3, pp. 566-569, 2006.

[11] S. Jarius, K. Ruprecht, B. Wildemann et al., "Contrasting disease patterns in seropositive and seronegative neuromyelitis optica: 
a multicentre study of 175 patients," Journal of Neuroinflammation, vol. 9, no. 1, article 14, 2012.

[12] M. Bradl, T. Misu, T. Takahashi et al., "Neuromyelitis optica: pathogenicity of patient immunoglobulin in vivo," Annals of Neurology, vol. 66, no. 5, pp. 630-643, 2009.

[13] M. Kinoshita, Y. Nakatsuji, T. Kimura et al., "Neuromyelitis optica: passive transfer to rats by human immunoglobulin," Biochemical and Biophysical Research Communications, vol. 386, no. 4, pp. 623-627, 2009.

[14] S. Saadoun, P. Waters, B. A. Bell, A. Vincent, A. S. Verkman, and M. C. Papadopoulos, "Intra-cerebral injection of neuromyelitis optica immunoglobulin $\mathrm{G}$ and human complement produces neuromyelitis optica lesions in mice," Brain, vol. 133, no. 2, pp. 349-361, 2010.

[15] T. Takahashi, K. Fujihara, I. Nakashima et al., "Anti-aquaporin4 antibody is involved in the pathogenesis of NMO: a study on antibody titre," Brain, vol. 130, no. 5, pp. 1235-1243, 2007.

[16] S. Jarius, F. Aboul-Enein, P. Waters et al., "Antibody to aquaporin-4 in the long-term course of neuromyelitis optica," Brain, vol. 131, no. 11, pp. 3072-3080, 2008.

[17] P. Waters, S. Jarius, E. Littleton et al., "Aquaporin-4 antibodies in neuromyelitis optica and longitudinally extensive transverse myelitis," Archives of Neurology, vol. 65, no. 7, pp. 913-919, 2008.

[18] S. H. Kim, S. M. Kim, A. Vincent et al., "Clinical characteristics, prognosis, and seropositivity to the anti-aquaporin-4 antibody in Korean patients with longitudinally extensive transverse myelitis," Journal of Neurology, vol. 257, no. 6, pp. 920-925, 2010.

[19] S. R. Hinson, A. McKeon, J. P. Fryer, M. Apiwattanakul, V. A. Lennon, and S. J. Pittock, "Prediction of neuromyelitis optica attack severity by quantitation of complement-mediated injury to aquaporin-4 -expressing cells," Archives of Neurology, vol. 66, no. 9, pp. 1164-1167, 2009.

[20] I. Dujmovic, S. Mader, K. Schanda et al., "Temporal dynamics of cerebrospinal fluid anti-aquaporin- 4 antibodies in patients with neuromyelitis optica spectrum disorders," Journal of Neuroimmunology, vol. 234, no. 1-2, pp. 124-130, 2011.

[21] S. Jarius, D. Franciotta, F. Paul et al., "Cerebrospinal fluid antibodies to aquaporin-4 in neuromyelitis optica and related disorders: frequency, origin, and diagnostic relevance," Journal of Neuroinflammation, vol. 7, article 52, 2010.

[22] I. De Vidi, G. Boursier, N. Delouche et al., "Strategy for antiaquaporin-4 auto-antibody identification and quantification using a new cell-based assay," Clinical Immunology, vol. 138, no. 3, pp. 239-246, 2011.

[23] S. R. Kalluri, Z. Illes, R. Srivastava et al., "Quantification and functional characterization of antibodies to native aquaporin 4 in neuromyelitis optica," Archives of Neurology, vol. 67, no. 10, pp. 1201-1208, 2010.

[24] L. Sabater, A. Giralt, A. Boronat et al., "Cytotoxic effect of neuromyelitis optica antibody (NMO-IgG) to astrocytes: an in vitro study," Journal of Neuroimmunology, vol. 215, no. 1-2, pp. 31-35, 2009.

[25] N. Collongues, R. Marignier, H. Zéphir et al., "Neuromyelitis optica in France: a multicenter study of 125 patients," Neurology, vol. 74, no. 9, pp. 736-742, 2010.

[26] I. Nakashima, T. Takahashi, B. A. C. Cree et al., "Transient increases in anti-aquaporin-4 antibody titers following rituximab treatment in neuromyelitis optica, in association with elevated serum BAFF levels," Journal of Clinical Neuroscience, vol. 18, no. 7, pp. 997-998, 2011.
[27] H. L. Pellkofer, M. Krumbholz, A. Berthele et al., "Long-term follow-up of patients with neuromyelitis optica after repeated therapy with rituximab," Neurology, vol. 76, no. 15, pp. 1310-1315, 2011.

[28] E. C. Klawiter, E. Alvarez, J. Xu et al., "NMO-IgG detected in CSF in seronegative neuromyelitis optica," Neurology, vol. 72, no. 12, pp. 1101-1103, 2009. 


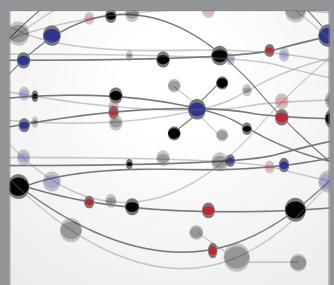

The Scientific World Journal
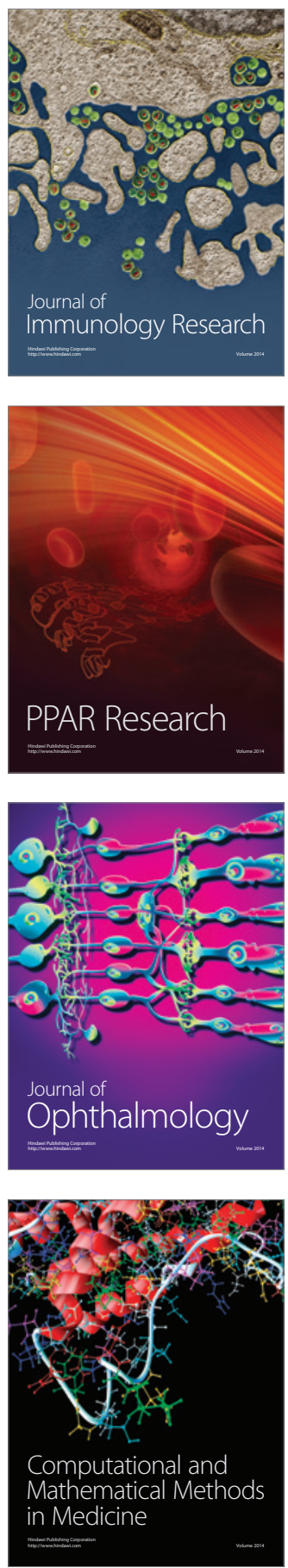

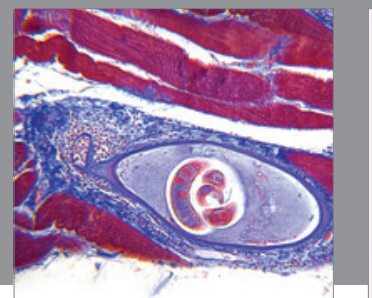

Gastroenterology

Research and Practice
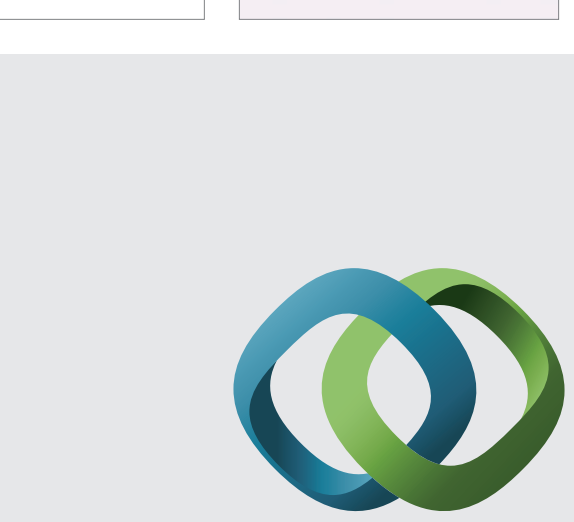

\section{Hindawi}

Submit your manuscripts at

http://www.hindawi.com
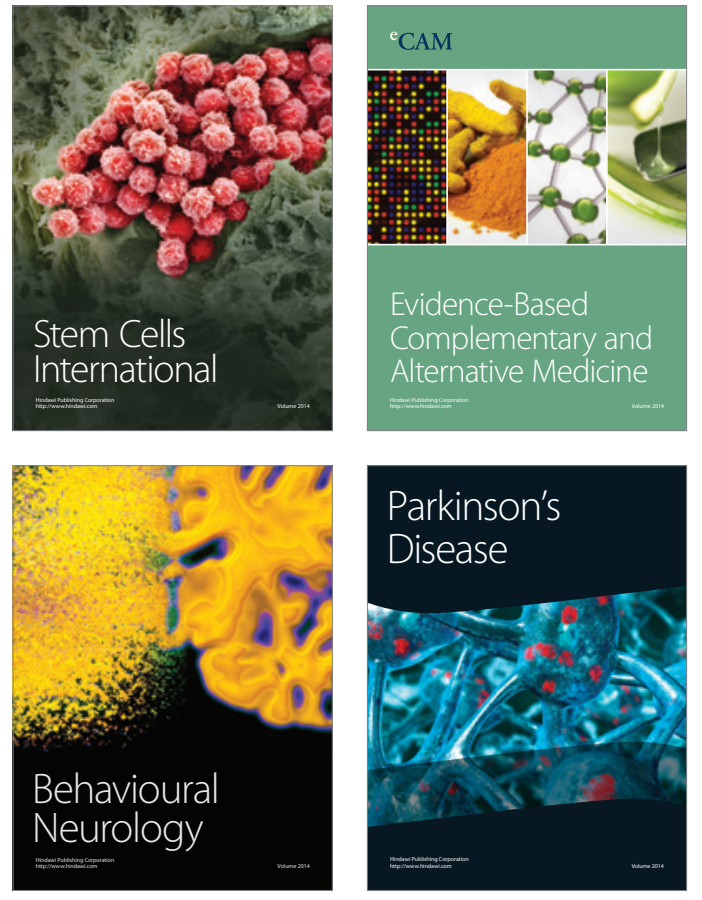
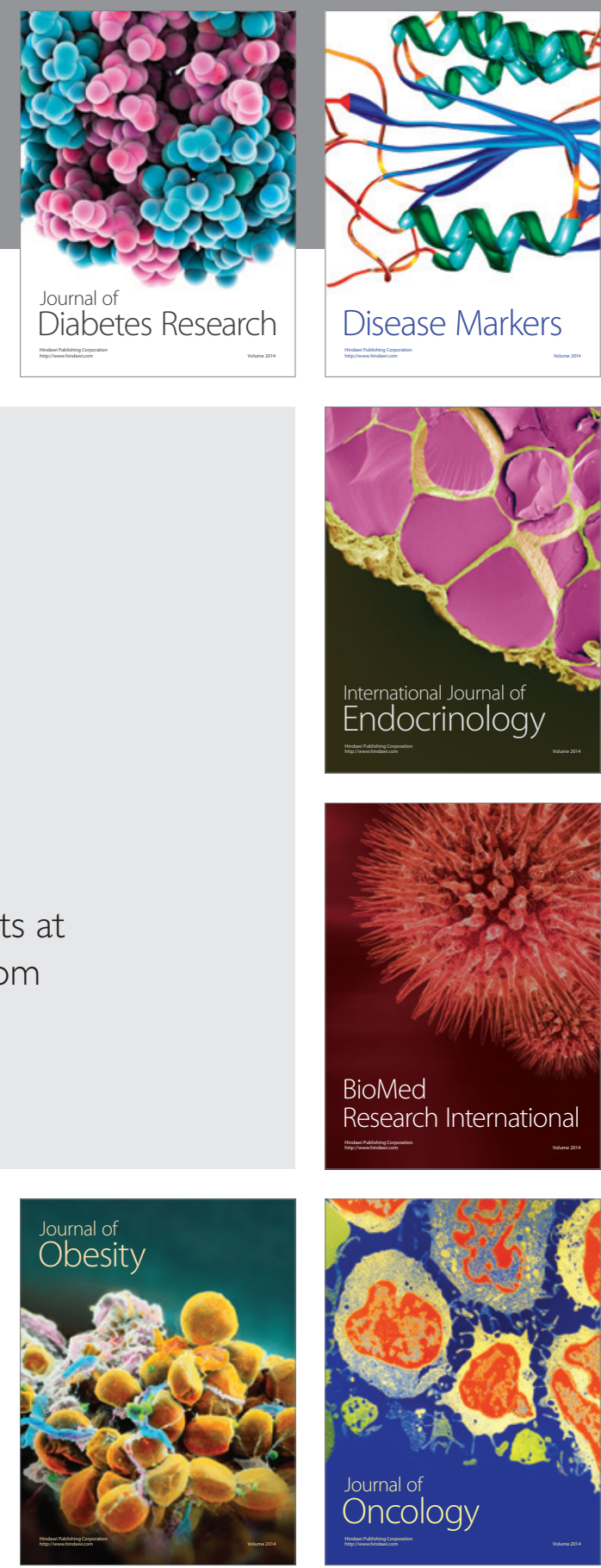

Disease Markers
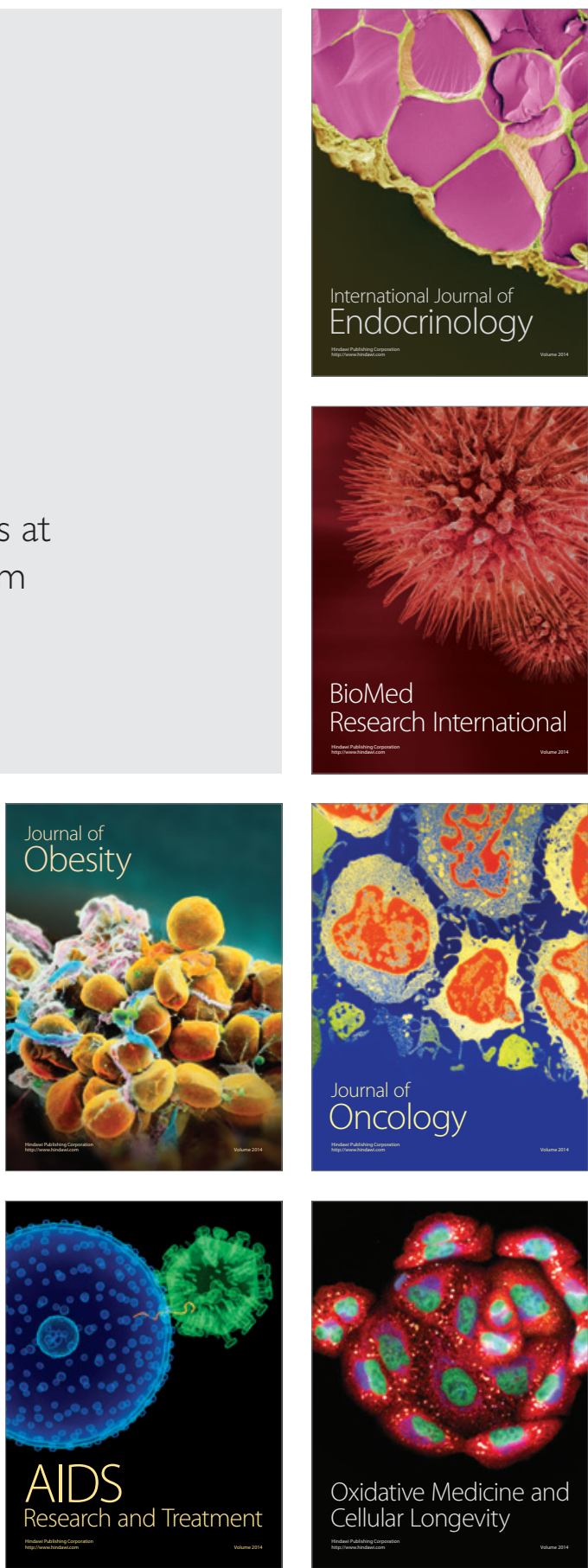